\title{
A tyrosine phosphorylation switch controls the interaction between the transmembrane modulator protein Wzd and the tyrosine kinase Wze of Lactobacillus rhamnosus
}

\author{
Hye-Ji Kang ${ }^{1,4}$, Christophe Gilbert ${ }^{2}$, Frédérique Badeaux ${ }^{1}$, Danièle Atlan ${ }^{3}$ and Gisèle LaPointe ${ }^{1 *}$
}

\begin{abstract}
Background: One proposed mechanism for assembly of secreted heteropolysaccharides by many Gram positive bacteria relies on the coordinated action of a polymerization complex through reversible phosphorylation events. The role of the tyrosine protein kinase transmembrane modulator is, however, not well understood.

Results: The protein sequences deduced from the wzb, wzd and wze genes from Lactobacillus rhamnosus ATCC 9595 and RW-9595 M contain motifs also found in corresponding proteins CpsB, CpsC and CpsD from Streptococcus pneumoniae D39 (serotype 2). Use of an anti-phosphotyrosine antibody demonstrated that both Wzd and Wze can be found in tyrosine phosphorylated form. When tyrosine 266 was mutated to phenylalanine, WzdY266F showed slightly less phosphorylated protein than those produced by using eight other tyrosine mutated Wzd genes, when expressed along with Wze and Wzb in Lactococcus lactis subsp. cremoris MG1363. In order to demonstrate the importance of ATP for the interactions among these proteins, native and fusion Wzb, Wzd and Wze proteins were expressed and purified from Escherichia coli cultures. The modulator protein, Wzd, binds with the phosphotyrosine kinase Wze, irrespective of its phosphorylation status. However, Wze attained a higher phosphorylation level after interacting with phosphorylated Wzd in the presence of $10 \mathrm{mM}$ ATP. This highly phosphorylated Wze did not remain in close association with phosphorylated Wzd.
\end{abstract}

Conclusion: The Wze tyrosine kinase protein of Lactobacillus rhamnosus thus carries out tyrosine phosphorylation of Wzd in addition to auto- and trans- phosphorylation of the kinase itself.

Keywords: Exopolysaccharide, Tyrosine phosphorylation, Co-polymerase, Transmembrane Modulator, Kinase, Lactobacillus rhamnosus

\section{Background}

Bacteria-host interactions are modulated by cell surface structures such as capsules and other extracellular polysaccharides (EPS). These polymers are generally considered to have a protective role against adverse environmental conditions [1]. Surface polysaccharides also play a role in recognition, and thus contribute to evading the host immune system by both pathogenic and commensal bacteria. The EPS from Lactobacillus

\footnotetext{
* Correspondence: gisele.lapointe@fsaa.ulaval.ca

'STELA Dairy Research Centre, INAF, Université Laval, Québec G1V 0A6, QC, Canada

Full list of author information is available at the end of the article
}

rhamnosus RW-9595 M stimulates interleukin, tumor necrosis factor and interferon gamma in mouse splenocytes [2], thus demonstrating a role in immunomodulation. Many strains of L. rhamnosus produce exopolysaccharides, but production levels vary greatly among strains. Strain RW-9595 M has shown the highest level of EPS production for a lactic acid bacterial species at $2000 \mathrm{mg} / \mathrm{L}$ under controlled $\mathrm{pH}$ conditions while strain ATCC 9595 produces a low level of EPS (116 mg/L) [3].

Polysaccharides secreted by Gram positive bacteria are synthesized by either a processive or a non-processive mechanism [4]. The Wzy-dependent non-processive mechanism requires the coordinated action of intracellular

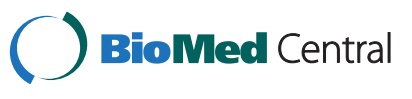

(c) 2015 Kang et al.; licensee BioMed Central. This is an Open Access article distributed under the terms of the Creative Commons Attribution License (http://creativecommons.org/licenses/by/4.0), which permits unrestricted use, distribution, and reproduction in any medium, provided the original work is properly credited. The Creative Commons Public Domain Dedication waiver (http://creativecommons.org/publicdomain/zero/1.0/) applies to the data made available in this article, unless otherwise stated. 
and membrane proteins with extracellular domains $[4,5]$. Inside the cell, glycosyltransferases use sugar nucleotides as substrates in order to assemble individual repeating units linked to a lipid carrier such as undecaprenylphosphate. When complete, each repeating unit is then transferred to the outer surface of the membrane, where the repeating units are assembled by a Wzy-dependent polymerization complex [4]. These polymers can then be covalently linked to the cell surface as capsules (CPS) in streptococci and staphylococci or released as exopolysaccharides (EPS) by lactic acid bacteria such as lactococci, streptococci or lactobacilli [5].

As components of the Wzy-dependent mechanism, the proteins proposed to be involved in determining the chain length of surface polysaccharides include a protein tyrosine phosphatase (PTP) and a polysaccharide co-polymerase (PCP) consisting of two domains, a cytosolic protein tyrosine kinase domain (PTK) and a tyrosine-protein kinase transmembrane activator or modulator (TKM) $[4,5]$. Polysaccharide co-polymerases have been classified into four subfamilies according to their sequence features [6]. The two domains are found in a single protein in Gram negative bacteria (subfamilies PCP1, PCP2a and PCP3), while they are coded by two separate proteins in Gram positive bacteria (PCP2b subfamily). In Streptococcus pneumoniae, three proteins with homologous functions named CpsB (PTP), CpsC (TKM) and CpsD (PTK) have been proposed to have roles in CPS polymerization and attachment [6]. Functional studies in S. pneumoniae have shown that tyrosine phosphorylation and dephosphorylation of CpsD have an impact on CPS production [7-9]. A stable complex consisting of $\mathrm{CpsB}, \mathrm{CpsC}, \mathrm{CpsD}$ and ATP is proposed to enhance capsule synthesis in S. pneumoniae strain D39 [8]. The level of phosphorylated CpsD (PTK) is positively correlated with capsule amount in strain D39 [7] and attachment of CPS to the cell wall is proposed to be enhanced by phosphorylated CpsD $[6,10]$. In this model, the transphosphorylation of the CpsD (PTK) requires the presence of the $\mathrm{CpsC}$ (TKM) and dephosphorylation of $\mathrm{CpsD}$ is carried out by the phosphatase CpsB (PTP), which can also inhibit the phosphorylation of CpsD. In addition to the role of facilitating CpsD phosphorylation, Morona et al. [6] have proposed that $\mathrm{CpsC}$ is also involved in attachment of CPS to the cell wall. Similar systems have been proposed for EPS elongation by Lactococcus lactis [11] and S. thermophilus [12]. Recent data suggests that the TKM/PTK complex plays a role in coordinating with cell wall growth by spatial regulation of capsule synthesis at the division septum in S. pneumoniae [13]. Furthermore, analogous chain length regulator protein Wzz is proposed to aid the Wzy polymerase by maintaining the nascent polysaccharide chain in a conformation conducive to continuing polymerization for O-antigen assembly [14].
The function of the complete polymerization complex and the precise role of each protein are not yet clearly understood, and may in fact differ among strains and species. When comparing the amino acid sequences between strains RW-9595 M and ATCC 9595, the Wzd tyrosine-protein kinase transmembrane modulator (TKM) and Wzb phosphatase (PTP) are 100\% identical, whereas the Wze kinase (PTK) of the two strains differ by one amino acid (T72 in ATCC 9595 versus K72 in RW-9595 M) [3]. Our previous work has shown the phosphatase activity of Wzb from L. rhamnosus [15]. In the present study, the purification of the three proteins (Wzb, Wzd, Wze) is described and their interactions are demonstrated in vitro. The impact of each tyrosine on the phosphorylation state of Wzd is revealed in vivo using point mutations and expressing these proteins in Lactococcus lactis subsp. cremoris MG1363. This work shows tyrosine phosphorylation of the Wzd modulator protein from $L$. rhamnosus and the impact of this phosphorylation on its interaction with the tyrosine protein kinase Wze.

\section{Methods}

\section{Bacterial strains and growth condition}

Bacterial strains used in this study are listed in Table 1. L. rhamnosus strains were grown without agitation at $37^{\circ} \mathrm{C}$ in MRS medium (Man Rogosa Sharpe Broth; EMD Chemicals Inc., Darmstadt, Germany) [16]. Escherichia coli strains were grown at $37^{\circ} \mathrm{C}$ in low salt Luria Bertani (LB) medium with agitation. L. lactis subsp. cremoris MG1363 was grown in M17 broth (Quelab, Montreal, Canada) supplemented with $0.5 \%(\mathrm{w} / \mathrm{v})$ glucose (GM17) incubated at $30^{\circ} \mathrm{C}$. For L. lactis transformants, chloramphenicol was added at $5 \mu \mathrm{g} / \mathrm{ml}$ and $100 \mu \mathrm{g} / \mathrm{ml}$ ampicillin was added for E. coli transformants.

\section{Plasmid construction and verification}

Plasmids used in this study are listed in Table 1 and oligonucleotide primers used are listed in Table 2. PCR was performed using standard conditions [20] with Taq Polymerase (Feldan-bio, Québec, Canada) and the primers listed in Table 1 specific for the $w z d, w z e$ and $w z b$ sequences from L. rhamnosus strain ATCC 9595 [GenBank: AY659976] and RW-9595 M [GenBank: AY659979] [3]. For studying in vitro protein interactions, the amplicons were ligated to the vectors PQE30 and pGEX-6-P and E. coli strains were transformed with the resulting recombinant plasmids by a standard electroporation procedure [21].

L. lactis subsp. cremoris MG1363 was used as host strain for the Wzy-dependent polymerization complex from L. rhamnosus RW-9595 M. A total of ten plasmids were constructed for expressing Wze and Wzb in conjunction with the wild type or nine mutated versions of the 
Table 1 Bacterial strains and plasmids

\begin{tabular}{|c|c|c|}
\hline Strain or plasmid & Relevant characteristic(s) & Source or reference \\
\hline \multicolumn{3}{|l|}{ Strains } \\
\hline L. rhamnosus ATCC 9595 & Low EPS-producing strain (116 mg/L) & ATCC $^{1}$ \\
\hline L. rhamnosus RW-9595 M & High EPS-producing strain (1611 mg/L) & Denis Roy ${ }^{2}$ \\
\hline $\begin{array}{l}\text { L. lactis subsp. cremoris } \\
\text { MG1363 }\end{array}$ & Plasmid free (Lac-Prt-) & {$[17]$} \\
\hline E. coli NM522 & Cloning host (supE thi-1 $\triangle\left(\right.$ lac-proAB) $\Delta(m c r B-h s d S M) 5$ (rK- mK-) [F' proAB lac $\left.\left.{ }^{9} Z \Delta M 15\right]\right)$ & Stratagene $^{1}$ \\
\hline E. coli BL21(DE3) & Expression host $\left(\mathrm{B} \mathrm{F}^{-}, \mathrm{ompT}, \mathrm{hsdS}\left(\mathrm{r}_{\mathrm{B}}^{-}, \mathrm{m}_{\mathrm{B}}^{-}\right), \mathrm{gal}, \mathrm{dcm}\right)$ & EMD Chemicals \\
\hline E. coli C41(DE3) & Expression host derived from BL21 & [18] \\
\hline \multicolumn{3}{|l|}{ Plasmids } \\
\hline pMG36CT & Cm, 3.7 kb, pWV01 replicon & \\
\hline pMG36EB & pMG36CT containing $1.6 \mathrm{~kb}$ Xbal-Aatll PCR amplicon (wze and wzb) from RW-9595 M & This study \\
\hline PDWTEB & pMG36EB containing 923 bp Sacl-Xbal PCR amplicon (wild type wzd) from RW-9595 M & This study \\
\hline pDY33FEB & pMG36EB containing 923 bp Sacl-Xbal PCR amplicon (Y33F mutated wzd) from RW-9595 M & This study \\
\hline pDY44FEB & pMG36EB containing 923 bp Sacl-Xbal PCR amplicon (Y44F mutated wzd) from RW-9595 M & This study \\
\hline pDY77FEB & pMG36EB containing 923 bp Sacl-Xbal PCR amplicon (Y77F mutated wzd) from RW-9595 M & This study \\
\hline pDY110FEB & pMG36EB containing 923 bp Sacl-Xbal PCR amplicon (Y110F mutated wzd) from RW-9595 M & This study \\
\hline pDY114FEB & pMG36EB containing 923 bp Sacl-Xbal PCR amplicon (Y114F mutated wzd) from RW-9595 M & This study \\
\hline pDY124FEB & pMG36EB containing 923 bp Sacl-Xbal PCR amplicon (Y124F mutated wzd) from RW-9595 M & This study \\
\hline pDY134FEB & pMG36EB containing 923 bp Sacl-Xbal PCR amplicon (Y134F mutated wzd) from RW-9595 M & This study \\
\hline pDY141FEB & pMG36EB containing 923 bp Sacl-Xbal PCR amplicon (Y141F mutated wzd) from RW-9595 M & This study \\
\hline pDY266FEB & pMG36EB containing 923 bp Sacl-Xbal PCR amplicon (Y266F mutated wzd) from RW-9595 M & This study \\
\hline PQE30 & His-tag fusion protein expression vector; $\mathrm{Cm}^{r}, \mathrm{Am}^{\mathrm{r}}$ & Qiagen ${ }^{1}$ \\
\hline pQE31 & His-tag fusion protein expression vector; $\mathrm{Cm}^{r}, \mathrm{Am}^{r}$ & Qiagen ${ }^{1}$ \\
\hline pGEX-6P-3 & GST fusion protein expression vector & Amersham Biosciences ${ }^{1}$ \\
\hline pGSTWze & 779 bp digested PCR fragment (wze) cloned into the BamHI-Xhol site of pGEX-6P-3 & GST-tag Wze; This study \\
\hline pQEWzd & 912 bp BamHI/Xhol digested PCR fragment (wzd) cloned into the BamHI-Sall site of pQE30 & His-tag Wzd; This study \\
\hline pQEWze & 819 bp digested PCR fragment (wze) cloned into the BamHI-HindllI site of pQE31 & native Wze; This study \\
\hline pQEWzb & 805 bp Sstl-Kpnl digested fragment (wzb) from pCRB cloned into the Sstl-Kpnl site of pQE31 & native Wzb; This study \\
\hline
\end{tabular}

${ }^{1}$ ATCC (American Type Culture Collection, Manassas, VA, USA); EMD chemicals (Gibbstown, USA); Qiagen S.A. (Courtaboeuf, France); Stratagene (LaJolla, CA, USA); Amersham Biosciences (Orsay, France).

${ }^{2}$ Original source: Denis Roy, INAF, Université Laval. [19].

wzd gene in L. lactis subsp. cremoris MG1363 (Table 1). First, the two genes $w z e$ and $w z b$ were amplified with primers wzeRXbaI/wzeF-wzb and wzbR-wze/wzbFAatII for the first PCR, and two amplicons were combined by PCR using wzeRXbaI as reverse primer and wzbFAatII as forward primer. The combined gene amplicon was cloned into pMG36CT to form pMG36EB. Nine separate tyrosine mutations to phenylalanine in the nucleotide sequence of wzd from RW-9595 M were constructed by gene synthesis (GenScript, USA). Each $\Delta w z d$ gene was amplified and then cloned into pMG36EB (pMG36CT $+w z e, w z b)$ and the resulting recombinant plasmids were separately transformed in L. lactis using the previously-published electroporation procedure [22]. The inserts of all final constructs in each host strain were confirmed by sequencing with an ABI Prism 3100 apparatus.
Production and purification of native and fusion proteins An overnight E. coli culture was diluted 100-fold with fresh LB broth supplemented with ampicillin, which was incubated at $37^{\circ} \mathrm{C}$ with shaking until $\mathrm{A}_{600}$ reached 0.5 to 0.6 (equivalent to 1 to $2 \times 10^{8} \mathrm{CFU}$ ). Induction was initiated by adding IPTG to $1 \mathrm{mM}$ and incubation continued for $3 \mathrm{~h}$ with shaking at $37^{\circ} \mathrm{C}$. Cells were centrifuged at $18000 \mathrm{X} \mathrm{g}$ for $20 \mathrm{~min}$ at $4^{\circ} \mathrm{C}$ and the pellet was suspended in $15 \mathrm{ml}$ STE buffer $(10 \mathrm{mM}$ Tris-HCl, pH 8.0, $150 \mathrm{mM} \mathrm{NaCl}$ and $1 \mathrm{mM}$ EDTA) containing $200 \mu \mathrm{g} / \mathrm{ml}$ lysozyme. After $10 \mathrm{~min}$ incubation on ice, the cells were sonicated $(40 \mathrm{~W}$, $5 \mathrm{~min}$ ) and then centrifuged at $18000 \mathrm{X} g$ for $20 \mathrm{~min}$ at $4^{\circ} \mathrm{C}$ [23]. The cell pellet was suspended in $2 \mathrm{ml}$ ST buffer (50 $\mathrm{mM}$ Tris, $300 \mathrm{mM} \mathrm{NaCl}, 5 \mathrm{mM} \mathrm{ZnCl} 2$ and $20 \mathrm{mM} \beta$-mercaptoethanol) containing $10 \%$ sarcosyl and incubated overnight at $4^{\circ} \mathrm{C}$ [24]. The resulting suspensions 
Table 2 Oligonucleotide primers

\begin{tabular}{|c|c|c|}
\hline Primer $^{1}$ & Sequence $\left(5^{\prime}\right.$ to $\left.3^{\prime}\right)$ & Gene target \\
\hline$\overline{\text { WzdRSacl }}$ & CGg agc tCA AGA GCA AAT TGA CCT TGC AC & wzd \\
\hline WzdFXbal & CGt cta gaT ACT TAA ACG CGT CTC CGG CTT CG & wzd \\
\hline WzeRXbal & GCG tct aga TTG AGG AGA AAA AAC ATG AAT TाT TC & wze \\
\hline WzeF-Wzb & ATA CTA TCT AAG CTC AAT ACT TAA ACG CGT CTC CGG & wze \\
\hline WzbR-Wze & ATA AAT AGC ATG CCT TAG ATA GTA TTG GAA GGG GAA C & $w z b$ \\
\hline WzbFAatll & CGg acg tCA TGA AAT TAG CAC TCG CAC AAC C & $w z b$ \\
\hline WzdSBaml & CGg gat $\subset C A$ TTG ACC TTG CAC GAC TT GG & $w z d$ \\
\hline WzdRXhol & CCG ctC gag TAC TTA AAC GCG TCT CCG GC & $w z d$ \\
\hline WzeSBaml & CGg gat CCT CAT TAG AGA AAA TाT TGC ATA GAC & wze \\
\hline WzeRXhol & CCG ctc gag GAC AGT TAG AAG CGC ATG CT & wze \\
\hline WzbSEcol & Gga att cGA TTG ATG TGC ATT GCC ATA TG & $w z b$ \\
\hline WzbRXhol & CCG ctC gag TAC CTT AAT ACC GCG ACA ACA AAC & $w z b$ \\
\hline WzeRhamS & CGg gat cca GAC GCG TाT AAG TAT TGA GGA G & wze \\
\hline WzeRhamR & CCC aag ctt TGA CAG TTA GAA GCG CAT GC & wze \\
\hline
\end{tabular}

${ }^{1}$ Restriction sites in primers are indicated in lower case letters.

of proteins were diluted 10 times with PBS (phosphatebuffered saline). The $20 \mathrm{ml}$ suspension of $\mathrm{His}_{6}-\mathrm{Wzd}$ was added to $1 \mathrm{ml} \mathrm{Ni}^{2+}$ nitrilotriacetic acid $\left(\mathrm{Ni}^{2+}-\mathrm{NTA}\right)$ agarose resin (Qiagen) with $20 \mathrm{mM}$ imidazole. Batch binding was carried out overnight $(18 \mathrm{~h})$ with gentle stirring at $4^{\circ} \mathrm{C}$. For GST-Wze, glutathione sepharose 4B (GE Healthcare Life Sciences) was utilized for purification. When needed to ensure complete dephosphorylation, a $0.3 \mathrm{ml}$ aliquot of resin-bound $\mathrm{His}_{6}$-Wzd was treated with $600 \mathrm{U}$ YOP (Yersinia tyrosine phosphatase, New England BioLabs) at $30^{\circ} \mathrm{C}$ for $3 \mathrm{~h}$, then inactivated by heat $\left(65^{\circ} \mathrm{C}\right.$ for $\left.1 \mathrm{~h}\right)$. YOP-treated and untreated aliquots $(0.3 \mathrm{ml})$ were then transferred to columns and washed 3 times with PBS containing $20 \mathrm{mM}$ imidazole.

For proteins without any tag (Wzb and Wze), the cell pellet from $100 \mathrm{ml}$ of induced culture was suspended in $15 \mathrm{~mL}$ PBS $(\mathrm{pH} 7.3)$ then incubated for 20 minutes at $25^{\circ} \mathrm{C}$ with $200 \mu \mathrm{g} / \mathrm{ml}$ lysozyme. Lysis was completed by two passages on a French Press $\left(138 \mathrm{MPa}, 6^{\circ} \mathrm{C}\right)$ followed by centrifugation at $18000 \mathrm{X} g 15 \mathrm{~min}$, then the pellet was washed three times with PBS containing 5\% Triton X-100 and $2 \mathrm{M}$ urea, followed by two washes with PBS. The washed pellet was suspended in $5 \mathrm{ml}$ PBS containing $2 \%$ sarcosyl then proteins were precipitated by adding $7.5 \mathrm{ml}$ acetone followed by centrifugation for $30 \mathrm{~min}$ at $18000 \mathrm{X} g$. Sarcosyl was eliminated by two washes with $2.5 \mathrm{ml} \mathrm{70 \%}$ ethanol then the pellet was suspended in $1 \mathrm{ml}$ PBS containing $5 \mathrm{mM} \mathrm{MgCl}$ and $1 \mathrm{mM} \mathrm{DTT}$.

A volume of $15 \mathrm{ml}$ of $L$. lactis subsp. cremoris cells grown to $5 \times 10^{8} \mathrm{CFU} / \mathrm{ml}\left(\mathrm{A}_{600}\right.$ of 0.65$)$ in GM17 was centrifuged at $20000 \mathrm{X} \mathrm{g}$ for $10 \mathrm{~min}$ at $4^{\circ} \mathrm{C}$. The pellet was suspended in $1 \mathrm{ml}$ TE buffer $(10 \mathrm{mM}$ Tris $(\mathrm{pH} 8.0)$ and $0.1 \mathrm{mM}$ EDTA) and mechanically disrupted in a mini beadbeater (BioSpec, Bartlesville, USA) with $1 \mathrm{~g}$ of $0.1 \mathrm{~mm}$ dia. glass beads (BioSpec, Bartlesville, USA) by two $30 \mathrm{~s}$ treatments with $1 \mathrm{~min}$ cooling on ice between two treatments [11]. Twenty microliters of cell extract was separated by SDS-PAGE.

\section{Protein-protein interaction assay}

The in vitro protein interaction was carried out by His-pull down assay. Either $1 \mathrm{ml}$ of purified Wze or $10 \mathrm{ml}$ of diluted cell lysate of GST-Wze with $20 \mathrm{mM}$ imidazole were added to an aliquot $(300 \mu \mathrm{l})$ of the resin-conjugated $\mathrm{His}_{6}$-Wzd and incubated overnight $(18 \mathrm{~h})$ with stirring at $4^{\circ} \mathrm{C}$. In duplicate samples, $10 \mathrm{mM}$ ATP (Sigma) was added before the overnight incubation. The protein mixtures were then transferred to a column and washed with PBS containing $20 \mathrm{mM}$ imidazole. In duplicate wash solutions, either $200 \mu \mathrm{M}$ or $10 \mathrm{mM}$ ATP was added. When necessary, elution of bound proteins was carried out with $1 \mathrm{M}$ imidazole.

\section{Western immunoblotting}

Proteins separated by SDS-PAGE were transferred by semi-dry electroblotting onto polyvinylidene difluoride membranes in triplicate to detect proteins with three different antibodies. Phosphotyrosine protein detection was carried out with primary antibodies: the membrane blots were incubated overnight at $4^{\circ} \mathrm{C}$ using a 1:1000 dilution of mouse monoclonal IgG anti-phosphotyrosine 4G10 antibody (Millipore). Detection of tagged protein with $\mathrm{His}_{6}$ or GST was carried out with primary antibodies using a 1:1000 dilution of mouse monoclonal $\operatorname{IgG}_{2 \mathrm{ak}}$ anti-histidine tagged antibody (Millipore) or mouse monoclonal IgG antiGST tag antibody (Millipore), respectively. All membranes 
were incubated for $1 \mathrm{~h}$ with 1:20000 dilution of secondary antibody (goat anti mouse IgG) coupled with horseradish peroxidase (HRP) (Millipore). Supersignal substrate (Thermo scientific) was used for HRP detection and visualised by exposure to Amersham Hyperfilm ECL (GE).

\section{Ethics statement}

No human subjects, human material, or human data were used in this study.

\section{Results and discussion}

Polymerization must be strictly controlled with respect to precursor availability and the energy necessary to form glycosidic bonds as well as to transport the units out of the cell, and finally to attach the polymers to the cell in the form of capsular material or to release them. Significant advancement has been made in understanding some interactions among components of the biosynthetic complex, but further work is needed to elucidate how these interactions promote the assembly of polysaccharides on the cell surface. In L. rhamnosus, three proteins may play a role in the control of EPS production, namely Wzb (phosphatase), Wzd (tyrosine protein kinase transmembrane modulator) and Wze (phosphotyrosine kinase). Details of comparative sequence analysis of Wzb, Wzd and Wze can be found in the Additional file 1 (Figure S1 and S2). In this study, the three genes from L. rhamnosus strain RW-9595 M were transferred to L. lactis subsp. cremoris MG1363 in order to discover the phosphorylation state of the three proteins in vivo in a Gram-positive expression system, as well as to demonstrate the importance of individual tyrosine residues to total Wzd phosphorylation and to the phosphorylation state of Wze. In order to examine protein interactions in vitro and their effect on phosphorylation, the three genes $w z b, w z d$ and $w z e$ were expressed in E. coli to obtain tagged and untagged proteins.

\section{Tyrosine phosphorylation of nine tyrosine-mutated Wzd proteins in L. lactis}

Nine mutated $w z d$ genes changing a different tyrosine codon to phenylalanine were expressed individually in concert with wze and $w z b$ from L. rhamnosus RW-9595 M in L. lactis subsp. cremoris MG1363 carrying one of the nine constructed plasmids (Table 1). As negative controls, there was no phosphorylated protein when extracts of $L$. lactis subsp. cremoris MG1363 with or without plasmid pMG36CT were probed with the anti-phosphotyrosine antibody (data not shown). All nine separate tyrosine mutated Wzd proteins $(33 \mathrm{kDa})$ were phosphorylated (Figure 1). For six mutants (Figure 1, Lanes 1 to 6), band density was approximately equivalent to the wild-type Wzd (ratio of 1 , as determined by ImageJ densitometry analysis). Two mutant proteins had slightly less band density than

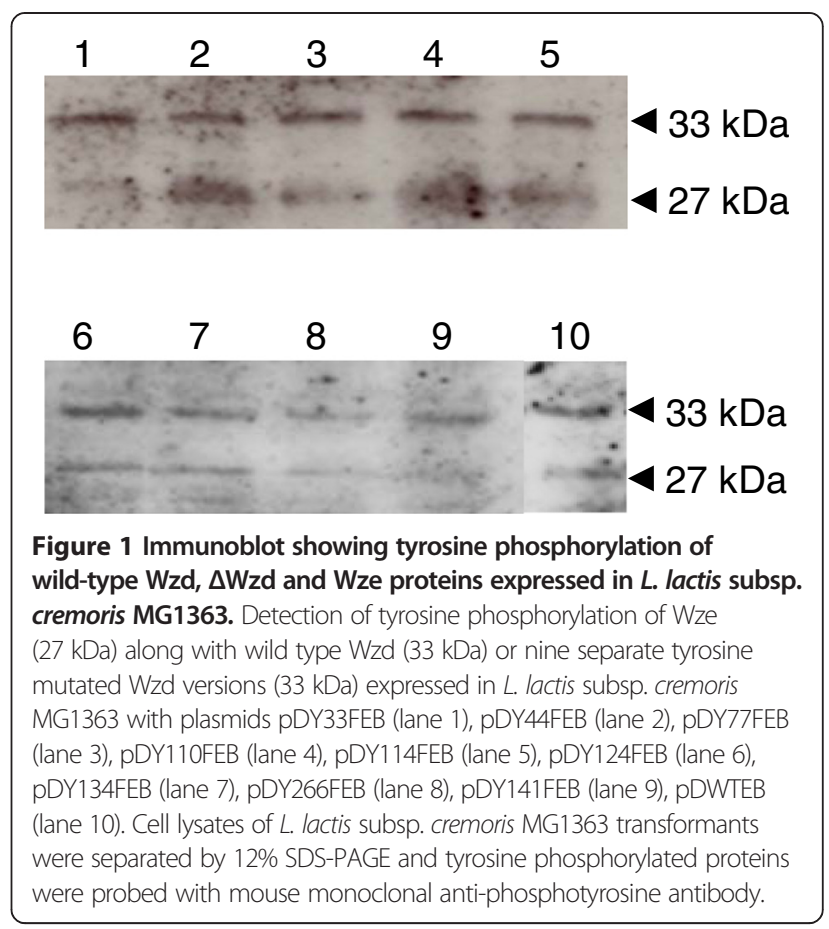

wild-type Wzd (Figure 1, Lane 10) with ratios of 0.9 (Wzd mutant Y134F; Lane 7) and 0.78 (Wzd mutant Y141F; Lane 9). The WzdY266F protein had the lowest ratio of 0.54 (Figure 1, lane 8) with respect to wild-type Wzd. Wze $(27 \mathrm{kDa})$ was also phosphorylated when co-expressed with each of the nine mutated Wzd proteins in L. lactis subsp. cremoris MG1363 (Figure 1), albeit to a lower level when Wzd carried mutations Y33F, Y266F and Y241F (Figure 1, Lanes 1, 8 and 9).

\section{Expression and purification of tagged and untagged proteins}

Overexpression of the $34 \mathrm{kDa} \mathrm{His}_{6}$-tagged Wzd protein from the wzd gene of L. rhamnosus ATCC 9595 was achieved in two E. coli strains, namely BL21(DE3) or C41(DE3) carrying pQEWzd (Figure 2A). Untagged Wzb and Wze proteins of the predicted molecular mass were obtained in E. coli strain NM522 carrying either pQEWzb (Figure 1B) or pQEWze. GST-tagged Wze protein was obtained using both $E$. coli strains C41(DE3) or BL21(DE3) carrying pGSTWze (Figure 2B).

\section{Tyrosine phosphorylation of $\mathrm{His}_{6}-\mathrm{Wzd}$ and GST-Wze expressed in E. coli}

The phosphorylation state of the expressed proteins was verified prior to testing protein interactions. To detect any autophosphorylation, proteins were incubated with or without ATP. As controls, cell lysates of the host strains BL21(DE3) and C41(DE3) carrying the vector plasmids did not show any tyrosine phosphorylated proteins, with or without added ATP (data not shown). In 

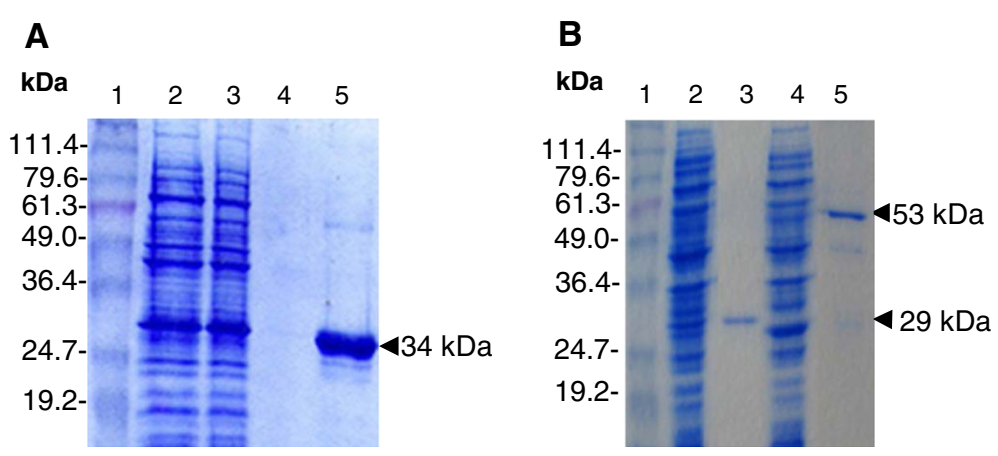

Figure 2 SDS-PAGE of IPTG-induced cell extracts and purified Wzd, Wzb and GST-Wze. (A) 34 kDa His - Wzd fusion protein purification by affinity chromatography from E. coli C41(DE3) (pQEWzd) IPTG-induced culture lysate. Lane 2: The flow through contains proteins not retained by the $\mathrm{Ni}^{2+}$ column. Lanes 3 and 4: fractions from first and last column washes, respectively; Lane 5: fraction from the elution step. (B) Wzb and GST-Wze purified from IPTG-induced culture lysates of E. coli NM522 (pQEWzb) and E. coli BL21(DE3) (pGSTWze). Lanes 2 and 4: lysed culture supernatant; Lanes 3 and 5: purified Wzb (29 kDa) and GST-Wze (53 kDa) proteins. Lane 1: BenchMark Prestained Protein Ladder (Invitrogen).

the absence of added ATP, the $\mathrm{His}_{6}$-Wzd purified from E. coli BL21(DE3) was recognized by the antiphosphotyrosine antibody, which shows tyrosine phosphorylation (Figure 3C). On the other hand, when purified after expression by strain C41(DE3), the similar amount of $\mathrm{His}_{6}$-Wzd was not phosphorylated after incubation either with or without ATP (Figure 3D), even though the protein was detected at by Western blot using the anti-His-tag antibody (data not shown). Under these conditions, the amount of phosphorylated $\mathrm{His}_{6}$-Wzd protein may be below the detection level of our assay.

The presence of GST-Wze was confirmed by anti-GST antibody and, as a control, GST expressed alone was not detected by the phosphotyrosine antibody (data not shown). The anti-phosphotyrosine antibody did detect phosphorylated GST-Wze in cell lysates of E. coli C41 (DE3) pGSTWze (Figure 3E) at the same level as in cell lysates of E. coli BL21(DE3) pGSTWze (data not shown) for the same amount of protein. The level of phosphorylated GST-Wze protein did not differ in the presence or absence of $10 \mathrm{mM}$ ATP. However, the untagged native Wze protein was not phosphorylated when produced by $E$. coli strains NM522 or BL21(DE3), whether or not ATP was present.

While GST-Wze was slightly phosphorylated in all $E$. coli host strains tested, the tyrosine phosphorylation of $\mathrm{His}_{6}$-Wzd depended on the strain of E. coli used for expression. The initial level of phosphorylation of these two proteins did not change when ATP was added. The cause of the difference between strain BL21(DE3) and its derivative mutant C41(DE3) has been attributed to a lower amount of T7 RNA polymerase in strain C41(DE3), which reduces transcription levels of cloned genes, actually leading to a more stable overexpression of recombinant proteins by strain C41(DE3) [25]. Phosphorylation of $\mathrm{His}_{6}$-Wzd may conceivably be carried out by a PTK of E. coli BL21(DE3), as was observed in studies of other proteins [26]. In our study, no other tyrosine-phosphorylated proteins were detected in the $E$. coli host strains used. UDP-glucose dehydrogenase YwqF from B. subtilis was phosphorylated by PTK Wzc from E. coli, showing that Gram-negative PTK is capable of phosphorylating proteins from Gram-positive species. Wze possesses a tyrosine in a similar position as the tyrosine 569 implicated in autophosphorylation of Wzc (PTK) in E. coli [27]. The autophosphorylation of tyrosine 569 did not occur without the N-terminal domain of Wzc, which is similar to the tyrosine kinase transmembrane modulator protein from Gram positive bacteria [28]. Thus, initial phosphorylation of GST-Wze implies that the presence of the GST tag may play a role as the modulator protein or that a protein of E. coli carries out tyrosine phosphorylation. Although serine phosphorylation of GST has been demonstrated previously [29], no tyrosine phosphorylation of GST itself was detected in our study (data not shown). GST may, however, possibly be phosphorylated by Wze. Nevertheless, there is no evidence of higher phosphorylation when GST-Wze is expressed alone, although the kinase is proposed to have autophosphorylation activity. This means that the Wzd modulator protein is required for higher levels of phosphorylation of the Wze tyrosine kinase, as described by Bender \& Yother [8]. To our knowledge, no previous study has detected the phosphorylation of the tyrosine kinase transmembrane modulator protein in any Gram-positive bacterial species.

\section{Interaction of $\mathrm{His}_{6}-\mathrm{Wzd}$ with Wzb and Wze}

Untagged $\mathrm{Wzb}$ and Wze were not retained by the nickel-charged affinity resin itself (data not shown), so they could be tested for affinity to resin-bound $\mathrm{His}_{6}$-Wzd. Wzb was not retained by $\mathrm{His}_{6}$-Wzd after washing the resin (data not shown), suggesting that no stable interaction was present between these two proteins. After incubating resin-bound $\mathrm{His}_{6}-\mathrm{Wzd}_{\mathrm{C} 41}$ and Wze together, the resin was divided into two fractions for 


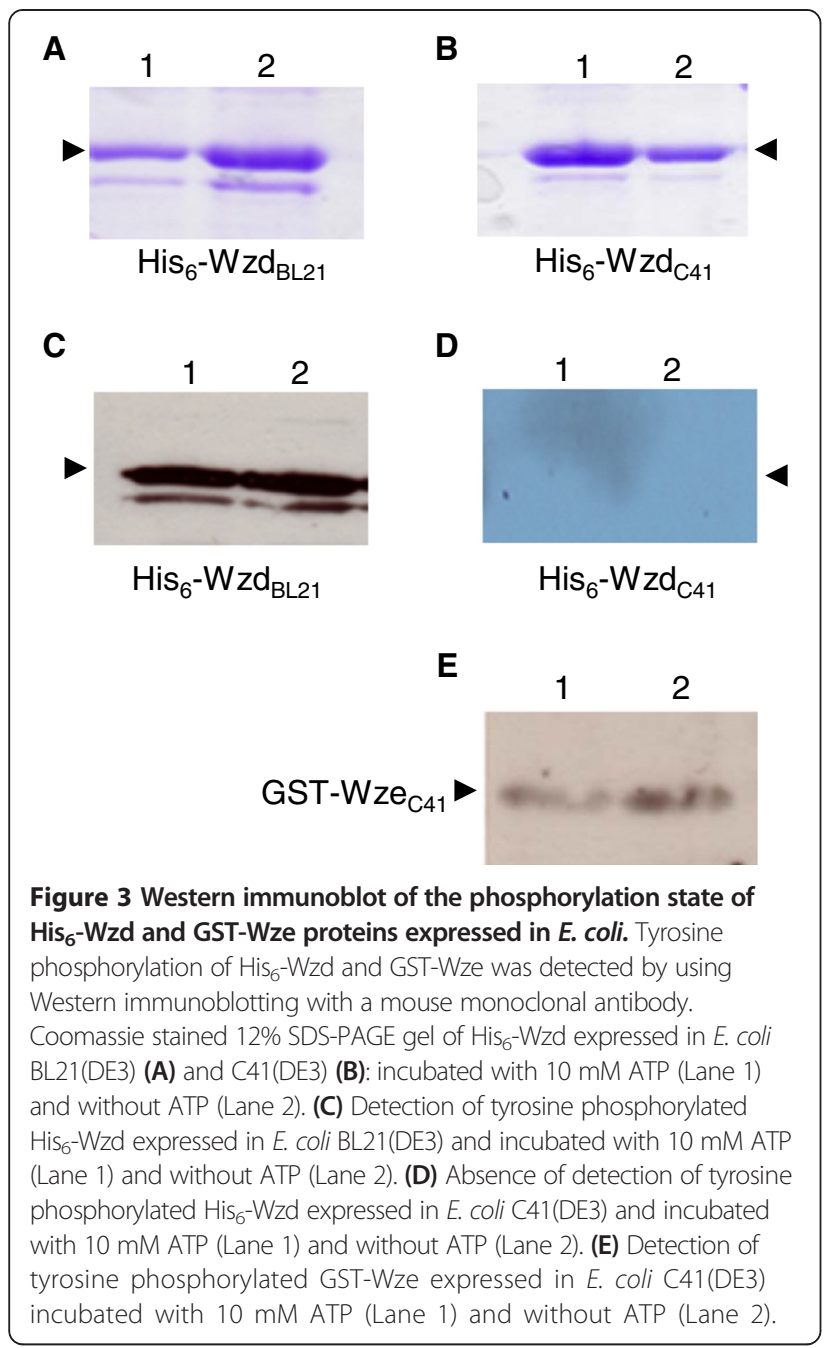

washing either with or without ATP. Wze was retained by $\mathrm{His}_{6}$-Wzd using the same washing conditions as with Wzb (Figure 4A). Without added ATP, the wash fraction did not contain any tyrosine-phosphorylated protein. However, when $200 \mu \mathrm{M}$ ATP was added to the washing solution, Wze protein was released from resin-bound $\mathrm{His}_{6}-\mathrm{Wzd}$ in each of three successive washing steps (Figure 4B, lanes 4, 5 and 6). After eluting the remaining bound proteins from the same column, only a small quantity of non-phosphorylated Wze was associated with $\mathrm{His}_{6}-\mathrm{Wzd}$ (Figure 4B, Lane 7). Moreover, using the anti-phosphotyrosine antibody, the Wzd protein was revealed to be tyrosine-phosphorylated after washing with $200 \mu \mathrm{M}$ ATP (Figure 4C).

GST-Wze alone also did not bind to the $\mathrm{Ni}^{2+}$ resin in the absence of $\mathrm{His}_{6}-\mathrm{Wzd}$ (data not shown). A cell lysate of $E$. coli C41(DE3) pGSTWze was passed through a column containing purified $\mathrm{His}_{6}$-Wzd expressed by either E. coli BL21(DE3) $\left(\mathrm{His}_{6}-\mathrm{Wzd}_{\mathrm{BL} 21}\right)$ or $\mathrm{C} 41(\mathrm{DE} 3)$ $\left(\mathrm{His}_{6}-\mathrm{Wzd}_{\mathrm{C} 41}\right)$ bound to the $\mathrm{Ni}^{2+}$ resin (Figure 5).

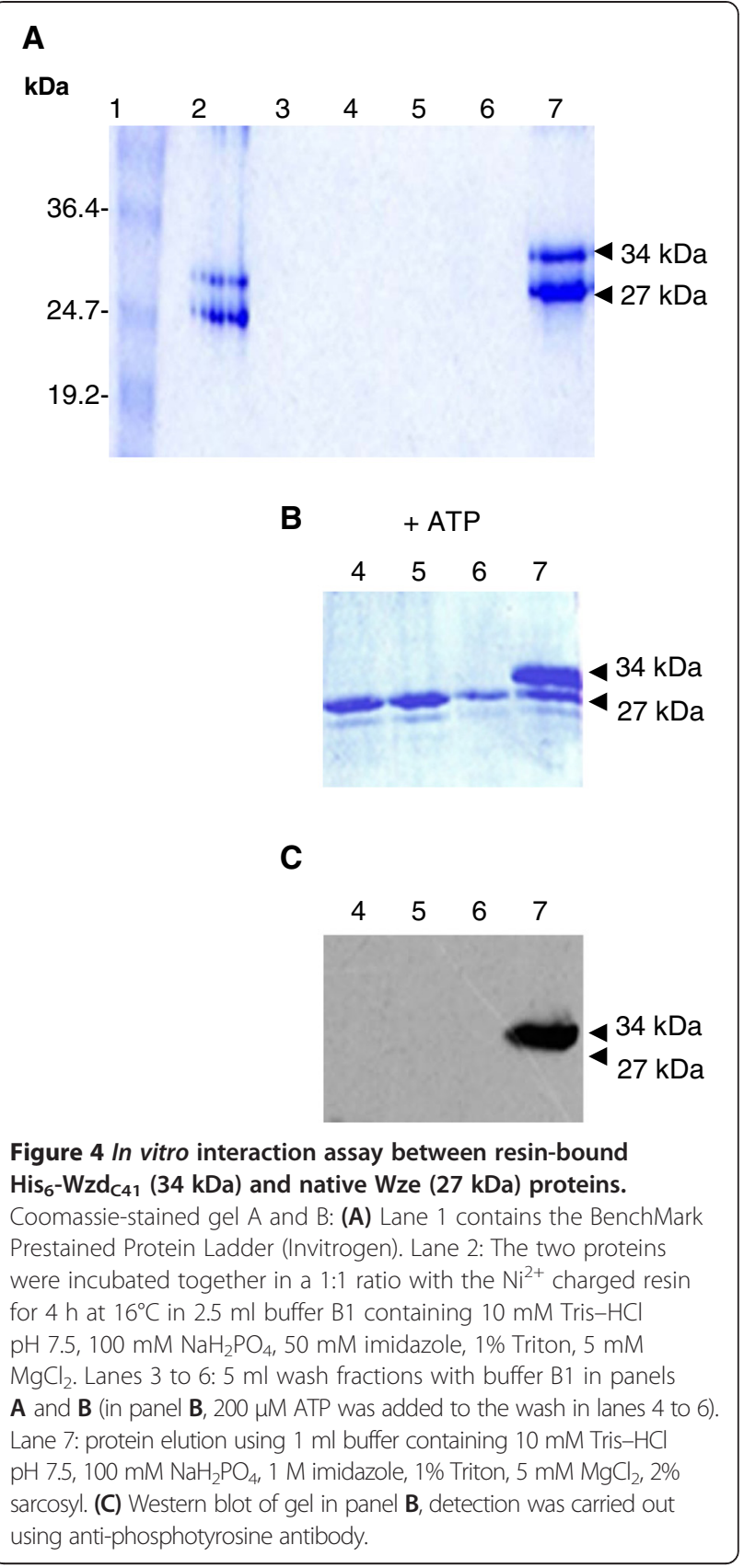

GST-Wze was retained by $\mathrm{His}_{6}-\mathrm{Wzd}_{\mathrm{BL} 21}$, both in the presence and absence of $10 \mathrm{mM}$ ATP during incubation and washing (Figure 5A). In the presence of ATP, there was no discernible change of tyrosine phosphorylation of $\mathrm{His}_{6}-\mathrm{Wzd}_{\mathrm{BL} 21}$ after the interaction (Figure 5B). GST-Wze was also retained by dephosphorylated $\mathrm{His}_{6}-\mathrm{Wzd}_{\mathrm{C} 41}$, in the presence and absence of $10 \mathrm{mM}$ ATP while YOP was retained as well (Figure 5C). YOP treatment did not affect protein migration and was not found in the wash fraction. In the presence or absence of ATP, $\mathrm{His}_{6}-\mathrm{Wzd}_{\mathrm{C} 41}$ on the resin with GST-Wze was not phosphorylated (Figure 5D). Bound GST-Wze showed slightly more phosphorylated 

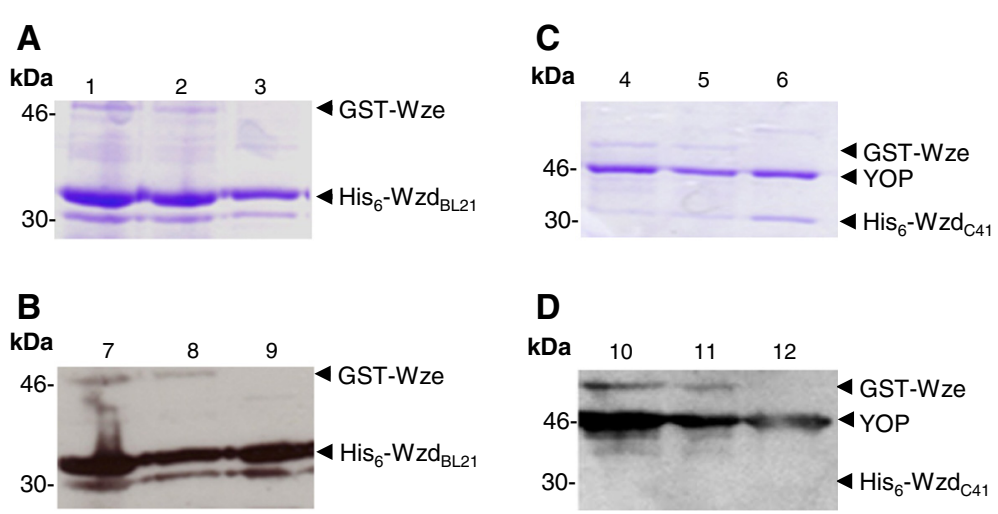

Figure 5 In vitro interaction assays between $\mathrm{His}_{6}-\mathrm{Wzd}(\mathbf{3 4} \mathrm{kDa})$ and GST-Wze $(53 \mathrm{kDa})$ proteins. His $-\mathrm{Wzd}_{6}$ bound to $\mathrm{Ni}^{2+}$ charged resin was incubated with the cell lysate of GST-Wze in the presence of $10 \mathrm{mM}$ ATP (lanes 1, 4, 7 and 10) or absence of ATP (lanes 2, 5, 8 and 11). Lanes 3, 6, 9 and 12 contain native $\mathrm{His}_{6}-\mathrm{Wzd}$ alone on the $\mathrm{Ni}^{2+}$ resin. Interaction of GST-Wze with His ${ }_{6}-\mathrm{Wzd}$ expressed by E. coli BL21(DE3): (A) Coomassie stained 12\% SDS-PAGE gel and (B) Western blot probed with mouse monoclonal anti-phosphotyrosine antibody. Interaction of GST-Wze with resin-bound $\mathrm{His}_{6}$-Wzd expressed by E. coli C41(DE3) and treated with YOP (Yersinia tyrosine phosphatase): (C) Coomassie stained 12\% SDS-PAGE gel and (D) Western blot probed with mouse monoclonal anti-phosphotyrosine antibody.

protein in the presence of ATP, but the difference does not appear significant and there was no discernible change in phosphorylation either before or after the interaction with $\mathrm{His}_{6}$-Wzd. The identical result was obtained using $\mathrm{His}_{6}-\mathrm{Wzd}_{\mathrm{C} 41}$ without YOP treatment and GST-Wze.

In contrast, the phosphorylation state of GST-Wze in the wash fractions was very different from that bound to $\mathrm{His}_{6}-\mathrm{Wzd}$ on the $\mathrm{Ni}^{2+}$ resin. For the same amount of protein (Figure 6A, lanes 1,2 and 3), GST-Wze was found in the wash fraction with a high phosphorylation level (Figure 6B, lanes 7, 8 and 9), after interacting with $\mathrm{His}_{6}-\mathrm{Wzd}_{\mathrm{BL} 21}$ in the presence of ATP. In the absence of ATP, the phosphorylated GST-Wze signal in the wash fraction was much lower (Figure 6B, lanes 10 to 12) for the same amount of protein (Figure 6A, lanes 4 to 6). There appears to be some phosphorylated GST-Wze retained by $\mathrm{His}_{6}-\mathrm{Wzd}$, but the highly phosphorylated GST-Wze did not bind strongly to Wzd. After interacting with $\mathrm{His}_{6}-\mathrm{Wzd}_{\mathrm{C} 41}$, GST-Wze was washed out of the resin, and the low level phosphorylation does not appear to significantly differ in the presence or absence of ATP (Figure 6C and D).

The results of this study reveal that no strong protein association was formed between Wzb and Wzd, while Wze interacts with phosphorylated as well as non-phosphorylated Wzd. When only slightly phosphorylated, GST-Wze did not affect the phosphorylation of Wzd, while Wzd was phosphorylated after interaction with non-phosphorylated Wze. This result shows that non-phosphorylated Wze is able to phosphorylate Wzd. In the presence of $10 \mathrm{mM}$ ATP, the unbound Wze was highly phosphorylated after interaction with phosphorylated Wzd, whereas Wze released from interaction with non-phosphorylated Wzd was not changed. The phosphorylation state of Wze retained with Wzd on the resin

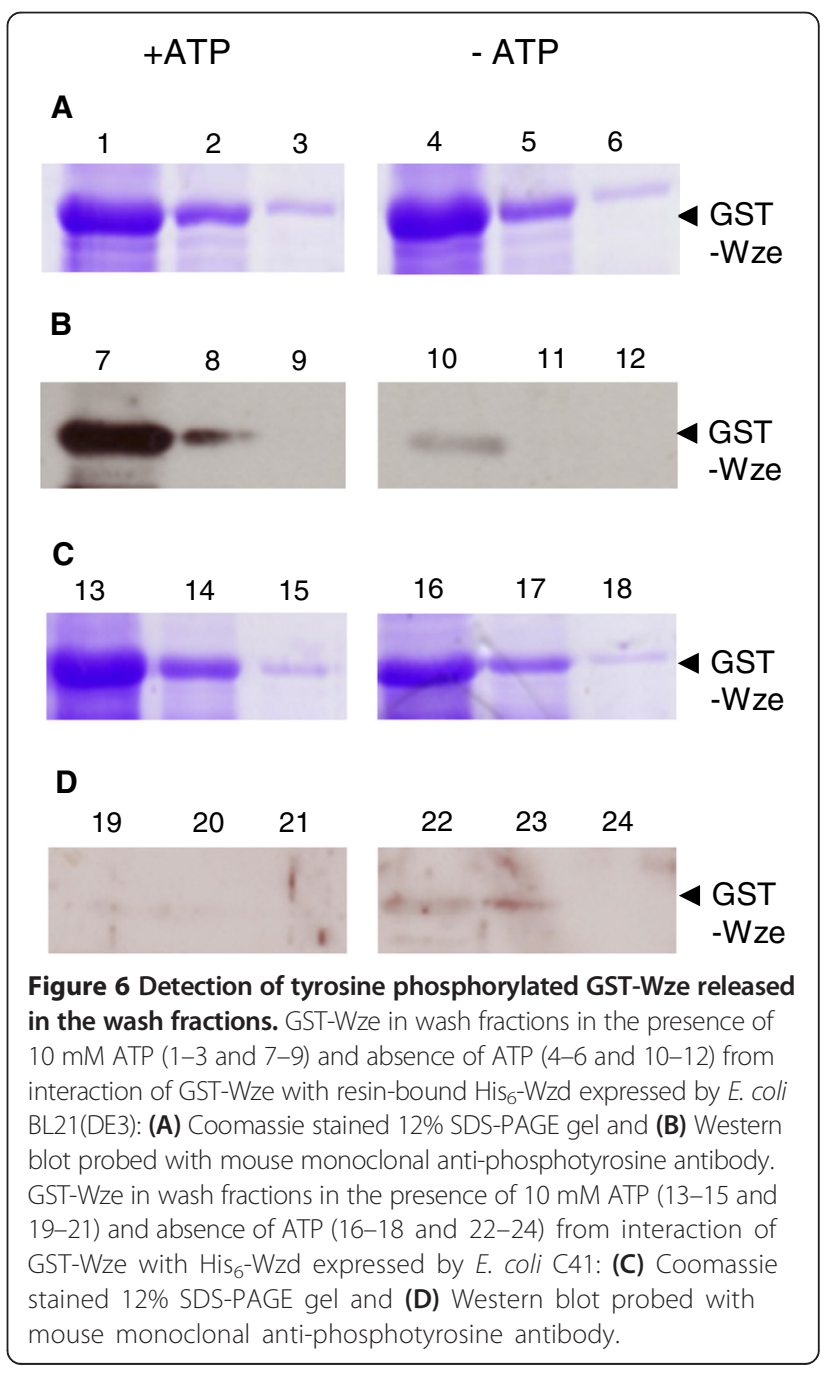


did not change after the interaction, regardless of the phosphorylation state of Wzd. This means that phosphorylated Wzd and ATP are necessary to attain a higher phosphorylation state of Wze and phosphorylated Wze does not remain bound in close association with phosphorylated Wzd.

Cefalo et al. [30] detected the interaction between Wzd (designated a transmembrane activator protein) and Wze (tyrosine kinase) in S. thermophilus and previous work done in S. pneumoniae [10] showed that CpsC/ CpsD (modulator/kinase) complex formation is necessary to detect tyrosine phosphorylated CpsD (tyrosine kinase). Our work shows evidence for this complex formation in L. rhamnosus and for tyrosine phosphorylation of both the kinase as well as the modulator protein. The results also support the role of Wzd as a modulator of the phosphorylation of the tyrosine kinase. EPS was not produced after deletion of EpsC (modulator) in S. thermophilus CNRZ1066 [12]. The Wzd modulator protein from $L$. rhamnosus could be involved in the production of EPS as proposed for EpsC. Indeed, several mutations occurring in the $\mathrm{CpsC}$ transmembrane modulator of a number of $S$. pneumoniae strains lead to defective cell wall attachment of CPS [6]. These include two tyrosine mutations in the $\mathrm{N}$-terminal region of $\mathrm{CpsC}$ (Y40C and Y83F) associated with a mucoid colony phenotype. In comparison with the L. rhamnosus Wzd sequence, equivalent tyrosine residues can be found in similar positions (Y33 and Y77) (see Additional file 1: Figure S2).
Wzd from L. rhamnosus has nine tyrosine residues and the last one (Tyr266) is located in the C-terminal cytoplasmic domain. The $w z d$, wze and $w z b$ genes from L. rhamnosus RW-9595 M were thus expressed in $L$. lactis subsp. cremoris MG1363 in order to verify which tyrosine residues could be involved in Wzd phosphorylation and whether Wze is then phosphorylated in vivo. While each of the nine separate tyrosine mutations led to Wzd phosphorylation, mutation of the C-terminal tyrosine (Tyr266) showed slightly weaker Wzd phosphorylation than most of the other eight mutations. This suggests that more than one tyrosine residue participates in Wzd phosphorylation, although Tyr266 appears to be slightly more important. In all cases, Wze was phosphorylated, although the phosphorylation levels of Wze were slightly lower when Wzd was mutated in positions Y33 (N-terminus), Y141 or Y266 (C-terminus). A previous study observed that the C-terminal cytoplasmic domain of CapA (transmembrane modulator) was essential for phosphorylation of CapB (kinase) in Staphylococcus aureus [31]. Tocilj et al. [32] proposed a role for the C-terminal Y191 tyrosine residue in alpha helix stabilization of the modulator, but no phosphorylation was proposed. Therefore, one or more of the mutated residues may be important for proper folding and membrane insertion of Wzd to allow good interaction with Wze, and thus facilitate the auto- or trans-phosphorylation of Wze.

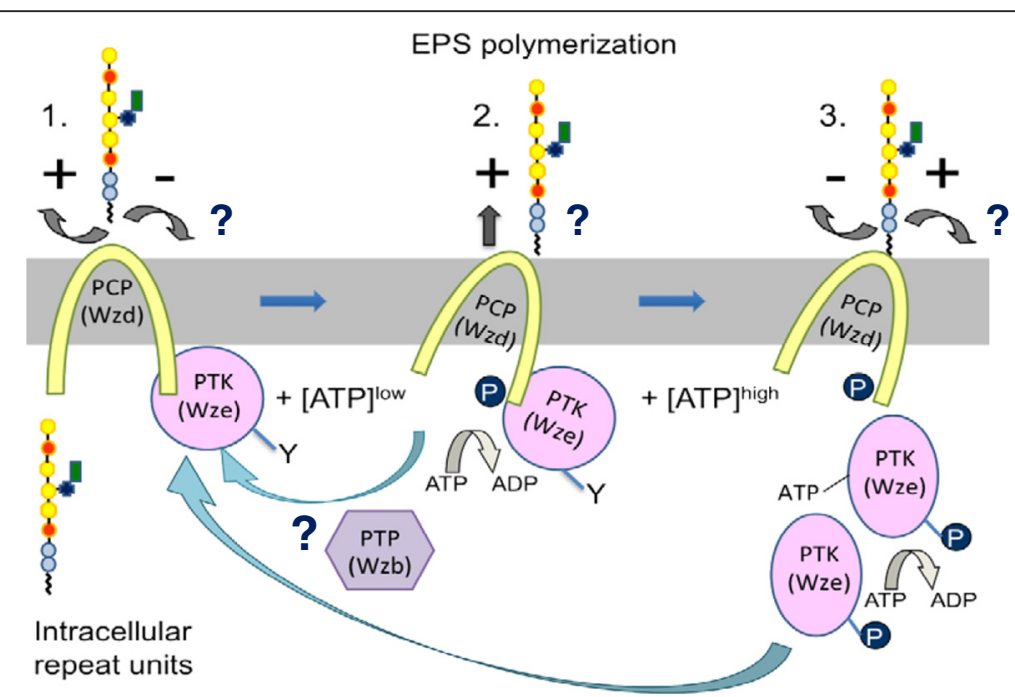

Figure 7 Model proposed for the tyrosine switch controlling EPS polymerization and attachment. 1. Non-phosphorylated Wzd and Wze interact by forming a complex leading to release of EPS (+) rather than attachment (-). $\mathbf{2}$. In the presence of ATP, Wze phosphorylates Wzd, allowing chain elongation by the polymerase $(+)$. This phosphorylation of Wzd destabilizes the protein interaction with Wze, allowing Wze to either bind with other non-phosphorylated Wzd proteins, or undergo auto- or trans-transphosphorylation. 3. A transitory interaction between phosphor-rylated Wzd and Wze in the presence of ATP is necessary for the phosphorylation of Wze, possibly allowing the attachment of polysaccharide to the cell wall (+). Phosphatase activity of Wzb for dephosphorylating Wzd and Wze would return the cycle to the non-phosphorylated Wzd/Wze complex that allows release of polysaccharide polymers (+). Question marks indicate proposed steps. 
In S. pneumoniae D39, when the cps $2 C$ (transmembrane modulator) was deleted, no capsule was detected, but low molecular weight products could be observed [9]. Defects in cell wall attachment were found when point mutations were introduced into $\operatorname{cps} 2 \mathrm{C}$, while some mutants were able to maintain wild type levels of total CPS production, including the Y82C mutation [9]. EPS were not produced at all when the equivalent modulator encoded by eps $A$ was deleted in L. lactis [11]. Cps2C from S. pneumoniae strain D39 contains 5 tyrosine residues while Cps2C from strain Rx1-19 F contains only 3, all located within the first $100 \mathrm{~N}$-terminal residues, as does the modulator of $S$. thermophilus and S. aureus. No tyrosine residue appears conserved among all 13 putative $\mathrm{PCP} 2 \mathrm{~b}$ sequences compared from staphylococci, streptococci, lactococci and lactobacilli, although the tyrosine positioned right after the first transmembrane domain is found in 12 out of the 13 sequences aligned. This indicates that the precise function of PCP2b could differ among species. The lactobacilli PCP2b sequences contain a supplementary stretch of 46 amino acids including 3 to 5 tyrosine residues that are not found in sequences from streptococci, lactococci and staphylococci. Wzd (L. rhamnosus), EpsA (L. lactis subsp. lactis or cremoris) and EpsB (L. johnsonii) have a total of 9, 7 and 6 tyrosine residues, respectively. The genera Lactobacillus and Lactococcus have common conserved tyrosines (both Y44 in the $\mathrm{N}$-terminus and Y266 in the C-terminal region) in their modulator protein sequences. These conserved tyrosines, especially the tyrosine located near the $\mathrm{C}$-terminal transmembrane region, may be associated with polymer extension and release instead of attachment. An equivalent C-terminal tyrosine 266 is absent from the $\mathrm{CpsC}$ sequences of $S$. pneumoniae, and $S$. thermophilus as well as the CapA sequence of $S$. aureus. Future work will need to focus on how these differences impact the production of capsular polysaccharides versus the production of released high molecular weight EPS.

\section{Conclusion}

Our study shows that the activity of Wzd is also modulated through tyrosine phosphorylation of more than one tyrosine residue, allowing the phosphorylation of Wze. This new information suggests that further modification of the model for the control of EPS elongation can be proposed (Figure 7). The non-phosphorylated complex may allow polysaccharides to be released instead of attached to the cell wall. When Wze phosphorylates Wzd, interaction of Wzd with the polymerase may promote polysaccharide elongation [14]. Destabilization of the protein interaction between Wzd and Wze liberates Wze to interact with other non-phosphorylated Wzd or Wze proteins. When Wzd is phosphorylated and
ATP is present, a transitory interaction allows Wze to autophosphorylate or transphosphorylate other Wze proteins, possibly slowing polymerization and leading to the attachment of polysaccharide to the cell wall if a ligase is present.

L. rhamnosus ATCC 9595 and RW-9595 M produce exopolysaccharides through a polymerase-dependent mechanism. Future experiments will focus on the role of the Wzb phosphatase in complex formation or dissociation, the relationship between the Wzd/Wze complex formation and EPS polymerization as well as the interaction of other proteins with this complex, including interactions of the polymerization complex with the proteins involved in synthesizing and transporting the repeat units. This approach will contribute to determining their function in modulating the biosynthesis of exopolysaccharides by $L$. rhamnosus.

\section{Additional file}

Additional file 1: Comparative sequence analysis of Wzb, Wzd and Wze. Figure S1. Comparison of transmembrane helix score plot of Wzd (A) from L. rhamnosus and CpsC19f (B) [GenBank: U09239]. Figure S2. Alignment of polysaccharide co-polymerases of thePCP2b subclass.

\section{Abbreviations}

EPS: Exopolysaccharide; CPS: Capsular polysaccharide; PTP: Protein tyrosine phosphatase; PTK: Protein tyrosine kinase; PCP: Polysaccharide co-polymerase; L rhamnosus: Lactobacillus rhamnosus; S pneumonia: Streptococcus pneumonia; S thermophilus: Streptococcus thermophilus; L lactis: Lactococcus lactis; $E$ coli: Escherichia coli.

\section{Competing interests}

The authors declare that they have no competing interests.

\section{Authors' contributions}

GL and CG conceived of the study with the participation of HK and DA in experimental design. GL and HK drafted the manuscript, as well as carried out initial sequence analysis. HK, CG and FB carried out the plasmid constructs and purification of fusion proteins as well as analyses of protein-protein interactions. GL, HK and CG participated in revising the manuscript. All authors confirm that they have read and approved the final manuscript.

\section{Acknowledgments}

This work was supported by a NSERC (Natural Sciences and Engineering Council of Canada) discovery grant awarded to GL.

\section{Author details}

15TELA Dairy Research Centre, INAF, Université Laval, Québec G1V 0A6, QC, Canada. ${ }^{2}$ CIRI-U1111 INSERM- UMR5308 CNRS-UCBL-ENSL, Université de Lyon, Université Lyon 1, 10 rue Dubois, bât. Lwoff, F-69622 Villeurbanne cedex, France. ${ }^{3}$ CNRS, UMR5240, Unité microbiologie, adaptation et pathogénie, Villeurbanne F-69622, France. ${ }^{4}$ Present address: Academy of Immunology and Microbiology (AIM), Institute for Basic Science (IBS), Pohang 790-784, Republic of Korea.

Received: 28 July 2014 Accepted: 4 February 2015

Published online: 21 February 2015

\section{References}

1. Ruas-Madiedo P, Hugenholtz J, Zoon P. An overview of the functionality of exopolysaccharides produced by lactic acid bacteria. Int Dairy J. 2002;12(2-3):163-71. 
2. Chabot S, Yu HL, De Léséleuc L, Cloutier D, Van Calsteren MR, Lessard M, et al. Exopolysaccharides from Lactobacillus rhamnosus RW-9595 M stimulate TNF. IL-6 and IL-12 in human and mouse cultured immunocompetent cells, and IFN-in mouse splenocytes. Lait. 2001;81(6):683-97.

3. Péant B, LaPointe G, Gilbert C, Atlan D, Ward P, Roy D. Comparative analysis of the exopolysaccharide biosynthesis gene clusters from four strains of Lactobacillus rhamnosus. Microbiology. 2005;151(6):1839-51.

4. Yother J. Capsules of Streptococcus pneumoniae and other bacteria: paradigms for polysaccharide biosynthesis and regulation. Annu Rev Microbiol. 2011;65:563-81.

5. De Vuyst L, De Vin F, Vaningelgem F, Degeest B. Recent developments in the biosynthesis and applications of heteropolysaccharides from lactic acid bacteria. Int Dairy J. 2001;11(9):687-707.

6. Morona JK, Morona R, Paton JC. Attachment of capsular polysaccharide to the cell wall of Streptococcus pneumoniae type 2 is required for invasive disease. Proc Natl Acad Sci U S A. 2006;103(22):8505.

7. Bender M, Cartee R, Yother J. Positive correlation between tyrosine phosphorylation of CpsD and capsular polysaccharide production in Streptococcus pneumoniae. J Bacteriol. 2003;185(20):6057-66.

8. Bender $M$, Yother J. CpsB is a modulator of capsule-associated tyrosine kinase activity in Streptococcus pneumoniae. J Biol Chem. 2001;276(51):47966-74.

9. Morona JK, Paton JC, Miller DC, Morona R. Tyrosine phosphorylation of CpsD negatively regulates capsular polysaccharide biosynthesis in Streptococcus pneumoniae. Mol Microbiol. 2000;35(6):1431-42.

10. Morona JK, Morona R, Miller DC, Paton JC. Mutational analysis of the carboxy-terminal $(\mathrm{YGX})_{4}$ repeat domain of $\mathrm{CpsD}$, an autophosphorylating tyrosine kinase required for capsule biosynthesis in Streptococcus pneumoniae. J Bacteriol. 2003;185(10):3009-19.

11. Groot N, Kleerebezem M. Mutational analysis of the Lactococcus lactis NIZO B40 exopolysaccharide (EPS) gene cluster: EPS biosynthesis correlates with unphosphorylated EpsB. J Appl Microbiol. 2007;103(6):2645-56.

12. Minic Z, Marie C, Delorme C, Faurie JM, Mercier G, Ehrlich D, et al. Control of EpsE, the phosphoglycosyltransferase initiating exopolysaccharide synthesis in Streptococcus thermophilus, by EpsD tyrosine kinase. J Bacteriol. 2007;189(4):1351-7.

13. Henriques MX, Rodrigues T, Carido M, Ferreira L, Filipe SR. Synthesis of capsular polysaccharide at the division septum of Streptococcus pneumoniae is dependent on a bacterial tyrosine kinase. Mol Microbiol. 2011;82(2):515-34.

14. Kalynych S, Valvano MA, Cygler M. Polysaccharide co-polymerases: the enigmatic conductors of the O-antigen assembly orchestra. Protein Eng, Des Sel. 2012:25(11):797-802.

15. LaPointe G, Atlan D, Gilbert C. Characterization and site-directed mutagenesis of Wzb, an O-phosphatase from Lactobacillus rhamnosus. BMC Biochem. 2008;9(1):10.

16. De Man J, Rogosa M, Sharpe ME. A medium for the cultivation of lactobacilli. J Appl Microbiol. 1960;23(1):130-5.

17. Gasson MJ. Plasmid complements of Streptococcus lactis NCDO 712 and other lactic streptococci after protoplast-induced curing. J Bacteriol. 1983;154(1):1-9.

18. Miroux B, Walker JE. Over-production of proteins in Escherichia coli: mutant hosts that allow synthesis of some membrane proteins and globular proteins at high levels. J Mol Biol. 1996;260(3):289-98.

19. Dupont I, Roy D, LaPointe G. Comparison of exopolysaccharide production by strains of Lactobacillus rhamnosus and Lactobacillus paracasei grown in chemically defined medium and milk. J Ind Microbiol Biotechnol. 2000:24(4):251-5

20. Sambrook J, Russell DW. Molecular cloning : A laboratory manual, 2nd ed. 2nd ed. New York USA: Cold Spring Harbor Laboratory Press; 1989.

21. Dower WJ, Miller JF, Ragsdale CW. High efficiency transformation of E. coli by high voltage electroporation. Nucleic Acids Res. 1988;16(13):6127-45.

22. Holo H, Nes IF. High-frequency transformation, by electroporation, of Lactococcus lactis subsp. cremoris grown with glycine in osmotically stabilized media. Appl Environ Microbiol. 1989;55(12):3119-23.

23. Zhuo Q, Piao J, Wang R, Yang X. Refolding and purification of non-fusion HPT protein expressed in Escherichia coli as inclusion bodies. Protein Expr Purif. 2005:41(1):53-60

24. Tao H, Liu W, Simmons BN, Harris HK, Cox TC, Massiah MA. Purifying natively folded proteins from inclusion bodies using sarkosyl, Triton X-100, and CHAPS. Biotechniques. 2010;48(1):61-4.
25. Wagner S, Klepsch MM, Schlegel S, Appel A, Draheim R, Tarry M, et al. Tuning Escherichia coli for membrane protein overexpression. Proc Natl Acad Sci U S A. 2008;105(38):14371-6.

26. Mijakovic I, Poncet S, Boël G, Mazé A, Gillet S, Jamet E, et al. Transmembrane modulator-dependent bacterial tyrosine kinase activates UDP-glucose dehydrogenases. EMBO J. 2003;22(18):4709-18.

27. Grangeasse C, Obadia B, Mijakovic I, Deutscher J, Cozzone AJ, Doublet P. Autophosphorylation of the Escherichia coli protein kinase Wzc regulates tyrosine phosphorylation of Ugd, a UDP-glucose dehydrogenase. J Biol Chem. 2003;278(41):39323-9.

28. Wugeditsch T, Paiment A, Hocking J, Drummelsmith J, Forrester C, Whitfield C. Phosphorylation of WzC, a tyrosine autokinase, is essential for assembly of group 1 capsular polysaccharides in Escherichia coli. J Biol Chem. 2001;276(4):2361-71.

29. Rodriguez P, Mitton B, Kranias EG. Phosphorylation of Glutathione-Stransferase by protein kinase C-a implications for affinity-tag purification. Biotechnol Lett. 2005;27(23-24):1869-73.

30. Cefalo AD, Broadbent JR, Welker DL. Protein-protein interactions among the components of the biosynthetic machinery responsible for exopolysaccharide production in Streptococcus thermophilus MR-1C. J Appl Microbiol. 2011;110(3):801-12.

31. Olivares-Illana V, Meyer P, Bechet E, Gueguen-Chaignon V, Soulat D, Lazereg-Riquier $S$, et al. Structural basis for the regulation mechanism of the tyrosine kinase CapB from Staphylococcus aureus. PLoS Biol. 2008;6(6):e143.

32. Tocilj A, Munger C, Proteau A, Morona R, Purins L, Ajamian E, et al. Bacteria polysaccharide co-polymerases share a common framework for control of polymer length. Nat Struct Mol Biol. 2008;15(2):130-8.

\section{Submit your next manuscript to BioMed Central and take full advantage of:}

- Convenient online submission

- Thorough peer review

- No space constraints or color figure charges

- Immediate publication on acceptance

- Inclusion in PubMed, CAS, Scopus and Google Scholar

- Research which is freely available for redistribution 